\title{
Orbital period modulation in close binaries due to cyclic $\alpha^{2}$-dynamo activity
}

\author{
G. Rüdiger ${ }^{1}$, D. Elstner ${ }^{1}$, A. F. Lanza ${ }^{2}$, and Th. Granzer ${ }^{1}$ \\ 1 Astrophysikalisches Institut Potsdam, An der Sternwarte 16, 14482 Potsdam, Germany \\ ${ }^{2}$ Osservatorio Astrofisico di Catania dell'Istituto Nazionale di Astrofisica, Via Santa Sofia 78, 95125 Catania, Italy
}

Received 10 April 2002 / Accepted 21 June 2002

\begin{abstract}
The question is answered whether dynamo-generated magnetic fields are able to produce such quadrupole terms in the gravity potential which can explain the observed cyclic orbital variation of RS CVn stars. We start with spherical dynamo models with outer convection zones but without any differential rotation, i.e. with $\alpha^{2}$-dynamos which are known as nonoscillating. With the known anisotropic $\alpha$-tensor of rapidly rotating stars the magnetic modes with the lowest dynamo numbers are nonaxisymmetric with a slow azimuthal drift. We also find, however, stable (i.e. with the lowest dynamo number) axisymmetric oscillating modes but only for a very special, highly inhomogeneous $\alpha$-tensor. The dynamo model is a linear one with an arbitrary field amplitude which can be scaled in order to reproduce the observations. The star proves to be as prolate during the maximum of the toroidal field energy and it proves to be as oblate during the maximum of the poloidal field energy. In the time average the influence of the toroidal field dominates and the star is slightly prolate. From the computed temporal variations of the gravitation quadrupole moment a magnetic field of more than $10^{5} \mathrm{G}$ is found in order to produce the period modulation of order $10^{-5}$ which has been observed.
\end{abstract}

Key words. magnetic fields - MHD - stars: individual: RS CVn

\section{Introduction}

The RS CVn systems defined by Hall (1976) are characterized by solar-like activity, the signatures of which have been observed in X-rays, UV, optical and radio spectral domains (see Linsky 1988; Rodonò 1992; Guinan \& Gimenez 1993; Strassmeier et al. 1993).

On the other hand, the eclipse times of the close binary systems of (say) $4 \ldots 5$ days varies by about $10 \mathrm{~s}$ with the time scale of the activity cycle (Fig. 1). The modulation time scale for RS CVn binaries goes from a few to several decades with a median value of around 40-50 yrs. The amplitude of the modulation is usually of the order of $\delta P / P \simeq 10^{-5}$ (Hall 1989, 1990).

The presence of a third body in the system, inducing a motion of the binary around a common centre of mass, with the related orbital period change, has been proposed as an explanation. However, in RS CVn systems, the mass derived for the third body is usually $1 \ldots 5 M_{\odot}$ and it is therefore very difficult to explain why such remarkable masses have not yet been detected.

Other possible causes of period modulation have therefore been suggested. Hall (1989) has shown that orbital period changes of alternate sign, in a sample of 101 Algols, are observed only in systems having a secondary of spectral

Send offprint requests to: G. Rüdiger, e-mail: gruediger@aip.de

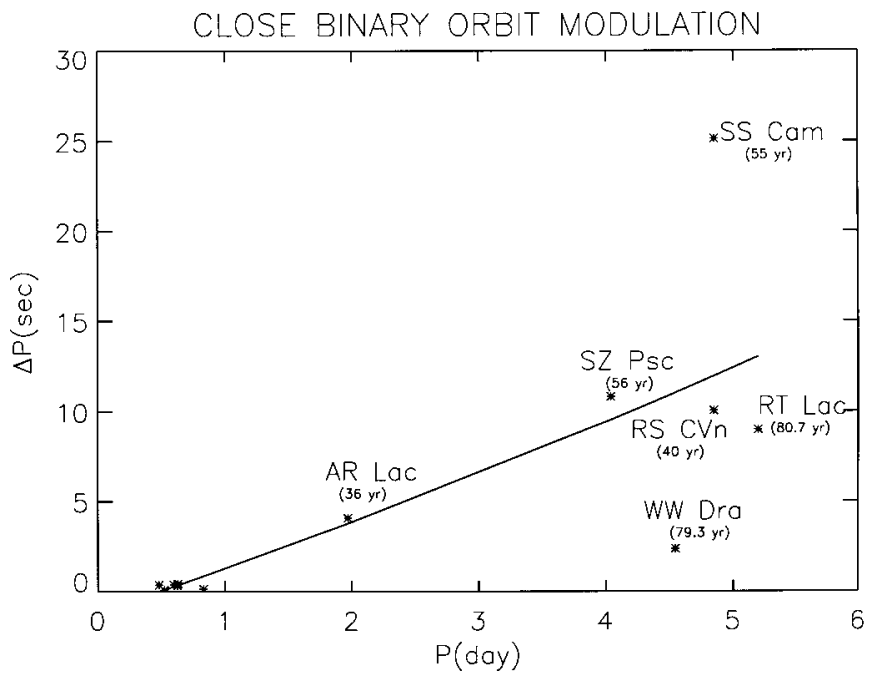

Fig. 1. The observed data for some considered close binary systems: period modulation, orbital period and magnetic cycle times (Lanza \& Rodonò 1999).

type F5 or later, i.e., those systems with components possessing deep outer convection zones. Systems with an early secondary typically exhibit monotone period changes. Such a result has recently been confirmed by Simon (1999) and Zavala et al. (2002). 
The possibility that a time-variable angular momentum loss, due to magnetic activity, be responsible for alternate orbital period changes was investigated by DeCampli \& Baliunas (1979). They concluded that this explanation was implausible because of the large mass loss required (about 3-4 orders of magnitude larger than allowed by observations) and also because of the long time scale needed to couple the variation of the stellar rotation to the orbital motion through tidal effects. The characteristic time scale for spin-orbit coupling in RS CVn binaries turns out to be of the order of $10^{3}$ years, i.e., about two orders of magnitude longer than the periods of the observed short-term modulations (Zahn 1989).

In view of such difficulties, Matese \& Whitmire (1983) proposed a new mechanism, based on a direct effect related to a cyclic change of the gravitational quadrupole moment of active stars. In this paper, we shall discuss the orbital period modulation under the hypothesis that a change of the gravitational quadrupole moment of active stars is responsible for the phenomenon based on an oscillating $\alpha^{2}$-dynamo (cf. Lanza et al. 1998).

After Lanza \& Rodonò (1999) the orbital modulation of close binaries is due to a modulation of the quadrupole moment $J_{2}$ of the gravitation potential of the active component, i.e.

$\frac{\delta P}{P}=-3\left(\frac{R}{a}\right)^{2} \delta J_{2}$

where $a$ is the semi-major axis of the orbit. Note that the orbital period decreases when the quadrupole moment increases and the star becomes more oblate ( $J_{2}$ increases).

The modulated quadrupole moment, in the present paper, may be assumed as to be due to the action of an oscillatory $\alpha^{2}$ dynamo. The typical value $a \simeq 4 R$ holds for RS CVn stars. If the potential series after Legendre polynomials,

$\phi=\phi_{0}+\phi_{2} P_{2}+\ldots$

is introduced, we find

$$
\frac{\delta P}{P}=3\left(\frac{R}{a}\right)^{2} \frac{R}{G M} \delta \phi_{2}(R)
$$

so that with the stellar model described below one obtains

$$
\frac{\delta P}{P} \simeq 4 \times 10^{-16} \delta \phi_{2}(R)
$$

with $\phi_{2}$ in cgs units. Positive (negative) $\phi_{2}(R)$ describe prolate (oblate) spheroids. The sign of the $\phi_{2}(R)$ follows from the fact that the gravitational potential of the sphere of radius $R$ is greater at the equator where the sphere is tangential to the oblate equipotential surface than at the pole where it is external. Since the Legendre polynomial $P_{2}(0)>P_{2}(\pi / 2), \phi_{2}(R)$ must be positive (negative) for prolate (oblate) configurations.

In order to obtain an effect of $\delta P / P \simeq 10^{-5}$ we thus have to find that magnetic field amplitude which produces a potential modulation of $\delta \phi_{2} \simeq 2.5 \times 10^{10} \mathrm{cgs}$. When the oblateness of the active star increases, $\phi_{2}$ becomes more negative and the orbital period decreases.

\section{Hydromagnetics}

\subsection{Basic equations}

Consider the equation system

$\nabla p=\rho \nabla \phi+L, \quad \triangle \phi=-4 \pi G \rho$

with the polytrope relation $p=K \rho^{\gamma}$ between pressure and density and the magnetic-induced Lorentz force $\boldsymbol{L}=\boldsymbol{J} \times \boldsymbol{B}$. Hence, from $(5)_{1}$

$\frac{\gamma K}{\gamma-1} \nabla \rho^{\gamma-1}=\nabla \phi+\frac{\boldsymbol{L}}{\rho}$

The quantities $p, \rho$ and $\phi$ may now be splitted such as

$p=p_{0}+p^{\prime}, \quad \rho=\rho_{0}+\rho^{\prime}, \quad \phi=\phi_{0}+\phi^{\prime}$,

where the primed quantities carry the influence of the magnetic acceleration $\boldsymbol{L} / \rho$. It follows from (6)

$\gamma K \nabla \psi^{\prime}=\nabla \phi^{\prime}+\frac{\boldsymbol{L}}{\rho_{0}}$

with the abbreviation $\psi^{\prime}=\rho_{0}^{\gamma-2} \rho^{\prime}$. Hence

$\gamma K \Delta \psi^{\prime}=-4 \pi G \rho_{0}^{2-\gamma} \psi^{\prime}+q$

with $q=\operatorname{div}\left(\boldsymbol{L} / \rho_{0}\right)$. All terms are of dimension $\mathrm{s}^{-2}$. Introducing $x=r / R$ we arrive at

$\Delta \psi^{\prime}+G^{*} \psi^{\prime}=q^{*}$

with

$G^{*}=\frac{4 \pi G R^{2}}{\gamma K} \rho_{0}^{2-\gamma}$

and

$q^{*}=\frac{q R^{2}}{\gamma K}$

Obviously, with $\gamma=5 / 3$ the profile $G^{*}$ is rather smooth while the $q^{*}$ must have a maximum in the bulk of the stellar interior. In Fig. 2 the main profiles are given for a stellar model of a subgiant with $M=2 M_{\odot}$ and $8.77 R_{\odot}$.

The resulting value for $K$ is

$K \simeq 4 \times 10^{15}$ cgs.

The Poisson equation reads

$\Delta \phi^{\prime}=-4 \pi G \rho_{0}^{2-\gamma} \psi^{\prime}$

where the profile $\rho_{0}^{2-\gamma}$ again is rather smooth.

According to the above considerations we are in particular interested in the coefficients of $P_{2}$ in the series expansion (2), so that the equation system which we have to solve reads

$\frac{\mathrm{d}^{2} \psi_{2}^{\prime}}{\mathrm{d} x^{2}}+\frac{2}{x} \frac{\mathrm{d} \psi_{2}^{\prime}}{\mathrm{d} x}-\frac{6}{x^{2}} \psi_{2}^{\prime}+G^{*} \psi_{2}^{\prime}=q_{2}^{*}$
$\frac{\mathrm{d}^{2} \phi_{2}^{\prime}}{\mathrm{d} x^{2}}+\frac{2}{x} \frac{\mathrm{d} \phi_{2}^{\prime}}{\mathrm{d} x}-\frac{6 \phi_{2}^{\prime}}{x^{2}}=-4 \pi G R^{2} \rho_{0}^{2-\gamma} \psi_{2}^{\prime}$. 


\subsection{Boundary conditions}

The equations must be integrated from $x=0$ where $\rho_{2}^{\prime}=$ $\psi_{2}^{\prime}=0$ up to the surface of the unperturbed model at $x=1$ where we also have used $\rho_{2}^{\prime}=0$ (see Appendix).

The Poisson equation $(15)_{2}$ must also be integrated from $x=0\left(\right.$ where $\left.\phi_{2}^{\prime} \propto x^{2}\right)$ to $x=1$ where $\mathrm{d} \phi_{2}^{\prime} / \mathrm{d} x+3 \phi_{2}^{\prime}=0$.

\subsection{The stellar model}

The mass and age of the stellar model considered is chosen to represent a typical RS CVn primary. In Table 1 the main properties of the model are listed. It was calculated from a fully convective, polytropic $(\gamma=5 / 3)$ start-model at a temperature of $T_{0}=4570 \mathrm{~K}$, luminosity $L_{0}=41.3 L_{\odot}$, a helium abundance of $Y=0.277$ and a metallicity of $Z=0.02$. Its quasi-static evolution to the main sequence and up to the subgiant phase has been calculated with an updated version of the Kippenhahn et al. (1967) stellar evolution code. Rotation was not taken into account. For the low-temperature regime, opacities of Alexander \& Ferguson (1994) were used to complement the OPAL96 opacities (Iglesias \& Rogers 1996). The OPAL equation-of-state (Rogers et al. 1996) was used whenever applicable, otherwise the MHD equation of state (Mihalas et al. 1990) was adopted. Local mixing-length theory was used for modeling the convection zone. The $\alpha_{\mathrm{MLT}}=l / H_{\mathrm{p}}$ parameter required by mixing-length theory was set to $\alpha_{\mathrm{MLT}}=1.67$. The nuclear reactions considered include the entire pp-chain and the CNO-cycle. The nuclear reaction rates and the energy generation rates are taken from Caughlan \& Fowler (1988). The inclusion of He-reactions is unnecessary due to the evolutionary stage of the star.

Table 1. Stellar parameters of the model considered. The age $t$ is given in Myrs. $M$ is the mass of the star, $L$ its luminosity, $R$ its radius, all in solar units. $T_{\text {eff }}$ is the effective temperature in K. $R_{\text {core }}$ is the fractional size of the radiative core. The central density $\rho_{\mathrm{c}}$ is in $\mathrm{g} / \mathrm{cm}^{3}$, while the central pressure, $p_{\mathrm{c}}$, is in dyn $/ \mathrm{cm}^{2}$.

\begin{tabular}{llllllll}
\hline \hline$t$ & $M$ & $L$ & $R$ & $T_{\text {eff }}$ & $R_{\text {core }}$ & $\rho_{\mathrm{c}}$ & $p_{\mathrm{c}}$ \\
\hline 934 & 2.0 & 35.4 & 8.77 & 4760 & 0.103 & 89.4 & $6.93 \times 10^{20}$ \\
\hline
\end{tabular}

\section{Dynamo theory}

The induction equation is

$\frac{\partial \boldsymbol{B}}{\partial t}=\operatorname{curl}\left(\alpha \circ \boldsymbol{B}-\eta_{\mathrm{T}} \operatorname{curl} \boldsymbol{B}\right)$.

We are here looking for a cyclic dynamo model with weak radial differential rotation. The differential rotation may be so weak that the dynamo behaves in its $\alpha^{2}$-regime. The question then is whether $\alpha^{2}$-dynamos are generally able to oscillate or not.

Rädler \& Bräuer (1987) suggested the possible existence of oscillating spherical $\alpha^{2}$-dynamos for outer convection zones of thickness smaller than $31 \%$ for $\alpha$ uniform throughout the whole
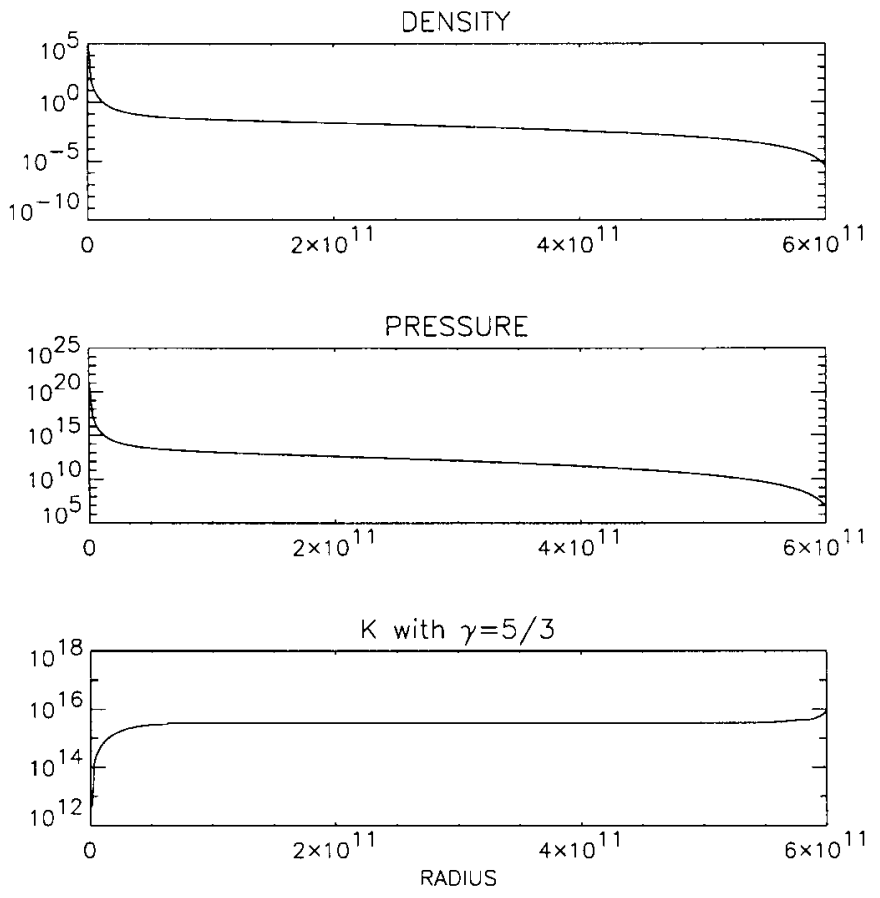

Fig. 2. The stellar model of a subgiant with $M=2 M_{\odot}$ and $8.77 R_{\odot}$. Note in particular the constancy of the polytrope factor $K=p \rho^{-5 / 3}$.

convection domain. The real situation is much more complicated. The $\alpha$-effect is antisymmetric with respect to the equator and it is strongly anisotropic insofar as the $\alpha_{z z}$-component differs even by sign from the $\alpha_{r r}$-component $\left(\alpha_{z z} \cdot \alpha_{\phi \phi}<0\right.$, Brandenburg et al. 1990; Ferrière 1992; Rüdiger \& Kitchatinov 1993). The immediate consequence of this situation is the existence of nonaxisymmetric modes (Rüdiger \& Elstner 1994; Moss \& Brandenburg 1995). Always, the nonaxisymmetric modes are drifting rather than oscillating. In the present paper the idea is followed whether more complicated $\alpha$-profiles exist (for the components of the anisotropic $\alpha$-tensor) for which the $\alpha^{2}$-dynamo indeed yields oscillating magnetic fields. The tendency of the latitudinal profiles which we use for the $\alpha$-effect is to concentrate it more and more towards the equator, i.e.

$\alpha \propto \sin ^{2 \lambda} \theta \cos \theta$

$(\lambda=0,1,2, \ldots)$ so that for higher $\lambda$ the dynamo design looks like a disk much more than a sphere. From Elstner et al. (1996) we know that indeed dynamos with anisotropic $\alpha$-effect and disk geometry exhibit oscillatory solutions. Also for disks with isotropic $\alpha$-effect oscillatory solutions have been presented (Baryshnikova \& Shukurov 1987; Rädler \& Bräuer 1987) the discussion of them, however, is beyond the scope of this paper. Our overall model represents a fast rotating star with outer convection zone so that the $\alpha$-effect is restricted to the region $x_{\text {in }} \leq x \leq 1$. Table 2 presents the eigenvalues of an $\alpha^{2}$ dynamo model with $x_{\text {in }}=0.5$. Always the lowest eigenvalue is written as boldface. The magnetic modes are symbolised as usual, i.e. A (S) gives dipolar (quadrupolar) geometry, the number $m=0,1,2 \ldots$ gives the azimuthal quantum number. 
Table 2. The dynamo numbers for isotropic $\alpha$-effect. $x_{\text {in }}=0.5$. The lowest eigenvalue for given $\lambda$ is printed in bold.

\begin{tabular}{lllll}
\hline \hline$\lambda$ & A0 & S0 & A1 & S1 \\
\hline 0 & 8.87 & $\mathbf{8 . 8 5}$ & 9.43 & 9.28 \\
1 & 28.6 (osc) & 28.5 (osc) & 26.7 & $\mathbf{2 6 . 1}$ \\
2 & 41.9 (osc) & $41.5($ osc $)$ & $\mathbf{3 8 . 5}$ & 38.6 \\
3 & 52.8 (osc) & 52.4 (osc) & $\mathbf{4 9 . 9}$ & 50.2 \\
\hline
\end{tabular}

Table 3. The dynamo numbers for anisotropic $\alpha$-effect $\left(\alpha_{z z}=-\alpha_{\phi \phi}\right)$, $x_{\text {in }}=0.5$.

\begin{tabular}{lllll}
\hline \hline$\lambda$ & A0 & S0 & A1 & S1 \\
\hline 0 & 21.9 (osc) & 21.8 (osc) & 14.3 & $\mathbf{1 4 . 2}$ \\
1 & 41.9 (osc) & 40.5 (osc) & 39.4 & $\mathbf{3 8 . 9}$ \\
2 & 59.7 (osc) & $\mathbf{5 7 . 1}$ (osc) & 58.8 & 58.3 \\
3 & 77.3 (osc) & $\mathbf{7 4 . 3}$ (osc) & 76.8 & 76.2 \\
\hline
\end{tabular}

The boldface modes are the excited stable eigenfunctions which differ for various $\lambda$ such as:

\begin{tabular}{lcccc}
\hline \hline$\lambda$ & 0 & 1 & 2 & 3 \\
\hline mode & S0 & S1 & A1 & A1 \\
\hline
\end{tabular}

Obviously, there are axisymmetric modes (for $\lambda=0$ in Eq. (17)) and nonaxisymmetric modes (for $\lambda>0$ in Eq. (17)) but oscillations do never occur.

For anisotropic $\alpha$-effect $\left(\alpha_{z z}=-\alpha_{\phi \phi}\right)$ there are, however, important differences (see Table 3 ). The excited modes are now:

\begin{tabular}{lcccc}
\hline \hline$\lambda$ & 0 & 1 & 2 & 3 \\
\hline mode & $\mathrm{S} 1$ & $\mathrm{~S} 1$ & $\mathrm{~S} 0(\mathrm{osc})$ & $\mathrm{S} 0(\mathrm{osc})$ \\
\hline
\end{tabular}

We find nonaxisymmetric field geometry for latitudinally spread $\alpha$-effect but for $\alpha$-effect concentrated in the equatorial region oscillatory and axisymmetric solutions occur. This situation remains unchanged for shallow convection zones but for deep convection zones the oscillatory modes disappear and are again replaced by drifting nonaxisymmetric modes (Rüdiger et al. 2002).

One always has to check the existence of all possible modes. The mode with the lowest dynamo number is the stable one. All our computations do not confirm the results by Weisshaar (1982) and Schubert \& Zhang (2000) who present oscillatory modes for much simpler models. They are probably not presenting the modes with the lowest eigenvalues, i.e. their solutions are unstable in the nonlinear regime (Krause \& Meinel 1988).

\section{Results}

The standard dynamo model with $x_{\text {in }}=0.5$ and with anisotropic $\alpha$-effect $\left(\alpha_{z z}=-\alpha_{\phi \phi}\right)$ for $\lambda=2$ is used in the following for the astrophysical applications. In units of the magnetic diffusivity in the convection zone the inner diffusivity $\left(x<x_{\text {in }}\right)$ is 0.01 (high temperature plasma) and the outer diffusivity $(x>1)$ is 10 (pseudovacuum).
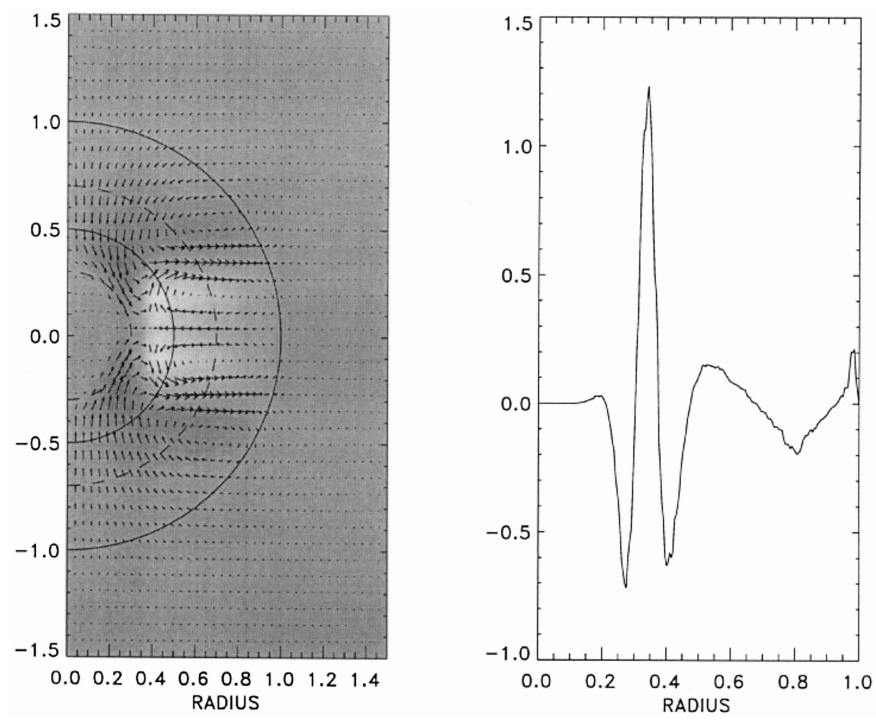

Fig. 3. Left: magnetic field configuration for the standard dynamo model with $x_{\text {in }}=0.5$ and with $\lambda=2$. The toroidal field structure is given by the grey-scale. The innermost circle represents the outer boundary of the perfectly conducting stellar core. Right: $q_{2}^{*}$ at timestep $0, q_{2}^{*}$ in cgs.
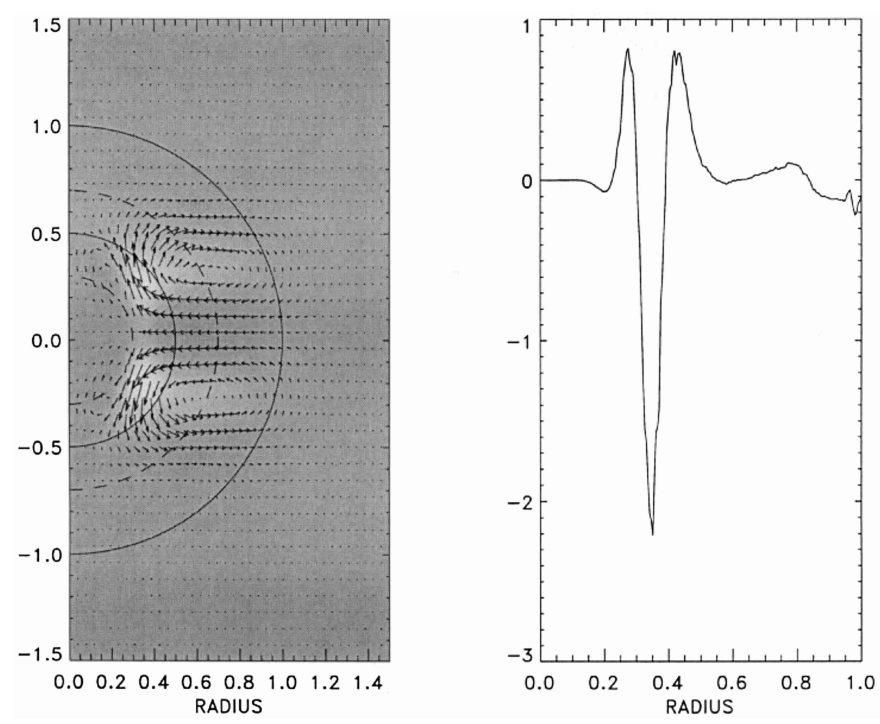

Fig. 4. The same as in Fig. 3 but for the timestep 0.2 diffusion time.

The cycle time from minimum to minimum proves to be 0.4 diffusion times. The Figs. 3-5 exhibit the magnetic fields at $\tau=0, \tau=0.2$ and $\tau=0.4$ covering a complete half-cycle. On the right-hand sides of the plots the Lorentz force divergence $q_{2}^{*}$ is given. It is similar in Figs. 3 and 5, but it differs in Fig. 4 by sign. The amplitude is always maximal below the convection zone. The magnetic pressure there has a source or a sink which yields magnetic-induced deformation of the star, if - as we do - any magnetic-induced internal flows are neglected. The resulting magnetic-induced deformation, therefore, can only be considered as a maximum and, on the other hand, the derived magnetic amplitudes must be considered as lower limits. 

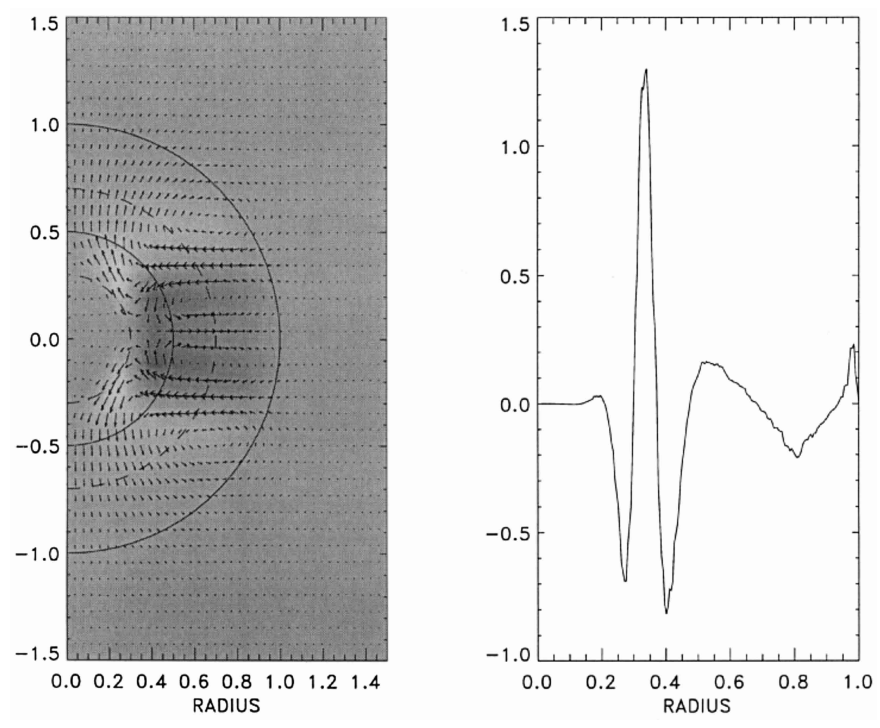

Fig. 5. The same as in Fig. 3 but for the timestep 0.4 diffusion time. Cycle time is 0.4 diffusion times.

In Fig. 6 the time series for the magnetic parameters are given, i.e. the magnetic energy which defines the cycle and the maximal field amplitudes. The latter are varying with amplitudes of order 0.5 . The variation is also reflected by the maximum of the Lorentz force (not shown) which finally, after the solution of the Eq. (15), yields the results plotted in Fig. 7. The $\phi_{2}$ at the stellar surface is positive and negative, i.e. the shape of the star varies between prolate and oblate. Figures 6 and 7 lead thus to the final result of our computations, i.e.

$\delta \phi_{2}(1) \simeq 0.4\left(\frac{B_{\max }}{0.5}\right)^{2} \simeq 1.6 B_{\max }^{2}$,

$B$ taken in Gauss. As one can take from Fig. 7 the star is prolate during the time of the magnetic maximum (of the toroidal field) and it is oblate during the time of the minimum. The latter behaviour has already been anticipated by Ferraro (1954) as the influence of the poloidal field. In the average over time the star appears to be prolate due to the influence of the toroidal field. This effect will be certainly amplified for stellar models with differential rotation as in this case the toroidal magnetic fields clearly dominate by orders of magnitude.

It is interesting to note that Eq. (18) can be compared with that obtained from consideration of the tensor virial theorem (cf. Lanza \& Rodonò 1999). Our detailed dynamo model basically confirms the connection between the quadrupole moment variation and the magnetic energy as indicated by that model.

\section{Discussion}

We have shown that a star with a deep convection zone can exhibit cyclic magnetic activity which may result in an axisymmetric deformation of the stellar sphere. No differential rotation was taken into account, the dynamo is a simple $\alpha^{2}$-dynamo. However, the cyclic solution only exists for highly anisotropic and inhomogeneous $\alpha$-effect. While the used anisotropy is well-established in theory and simulation, the high concentration of the $\alpha$-effect towards the equator (which was necessary for the oscillatory solution) is a speculation.
In order to compute the deformation of the star we have neglected the whole system of magnetic-induced internal flows. As this is certainly not completely correct, the resulting quadrupole moment must be considered as a maximal value.

With (4) and (18) we find

$$
\frac{\delta P}{P} \simeq 6.4 \times 10^{-16} B_{\max }^{2},
$$

so that a magnetic amplitude of

$B_{\max } \simeq 125 \mathrm{kG}$

is required to produce an orbit variation with $\delta P / P \simeq 10^{-5}$.

The question arises for the stability against buoyancy of such strong magnetic fields. Although there is, of course, no final answer to this question we want to stress that the result is fully consistent with the advection-computations within the frame of the second order correlation approximation. It is shown by Kitchatinov \& Rüdiger (1992) that for very strong magnetic fields one of the both advection phenomina (the "diamagnetism") disappears while the other one (the "turbulent buoyancy") survives and it goes downwards. In nonlinear dynamo models this effect must also be involved in the simulations but here, considering kinematic dynamos, this argument only suggests selfconsistency. Note that the turbulent buoyancy only exists in the mean-field concept but this is also true for the $\alpha$-effect ${ }^{1}$.

Our model, of course, only forms an example in order to simulate the real situation. We started the presented new application of stellar dynamo theory with three serious approximations. The first one concerns the stellar model because of its high values of mass and radius. It is not easy to discuss the consequences of variations of mass and radius. What we present here is one of the possible examples. Probably more serious is the neglect of any differential rotation and/or the ignorance of the off-diagonal elements in the $\alpha$-tensor. Diamagnetism, turbulent buoyancy and longitudinal pumping have strong implications for the temporal character of the dynamo. The presented highly simplified dynamo model is only an example. Again it is not easy to estimate the influences of the neglected turbulence-induced phenomena. In particular, all the $\alpha \Omega$-dynamos differ from $\alpha^{2}$-dynamos by the highly different amplitudes of toroidal and poloidal fields. There should, of course, result modifications for the magnetic-induced gravity quadrupole moment and the resulting minimum magnetic field necessary for the observation of orbital modulations.

Also the last of our simplifications seems to be a serious one and this is the complete ignorance of internal flows in our model. As known, a dynamo can saturate by inducing large-scale poloidal flow patterns (Malkus \& Proctor 1975) as well cyclic variations of the rotation law (Schüssler 1981; Yoshimura 1981). One can thus also argue with Applegate (1992) that the centrifugal force must be included into Eq. (5) in order to remain self-consistent. As known from the theory of the solar torsional oscillations then one has to solve the time-dependent equation for the angular momentum. With the

\footnotetext{
${ }^{1}$ More exactly speaking, it is part of the $\alpha$-effect.
} 

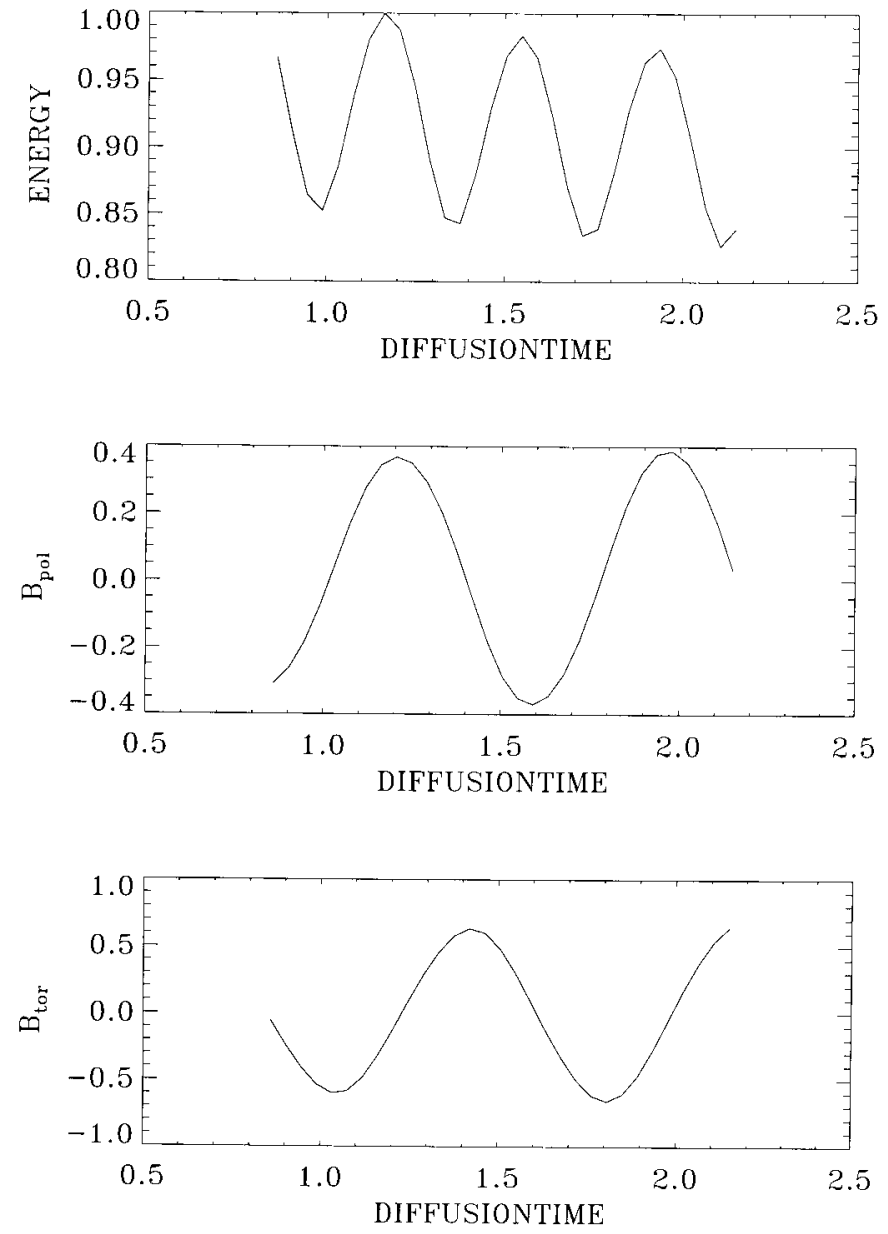

Fig. 6. Time series for important magnetic parameters. Top: total magnetic energy, Middle: radial magnetic field component at $x=0.25$ and in the equatorial plane, Bottom: toroidal magnetic field component at $x=0.25$ and in the equatorial plane. The cycle time is 0.4 .

present paper we have only done here a first step to bring together dynamo theory computations with observations in close binary systems.

Acknowledgements. The numerical assistance of Y. Zhang during his stay in Potsdam is acknowledged by the authors.

\section{Appendix A:}

If the Lorentz force can be neglected near the outer boundary the momentum equation is

$\nabla p=\rho \nabla \phi$

which with $p=K \rho^{\gamma}$ becomes

$K \gamma \rho^{\gamma-2} \nabla \rho=\nabla \phi$

This equation implies that $\rho=\rho(\phi)$ and that

$\frac{\mathrm{d} \rho}{\mathrm{d} \phi}=\frac{[\rho(\phi)]^{2-\gamma}}{K \gamma}$

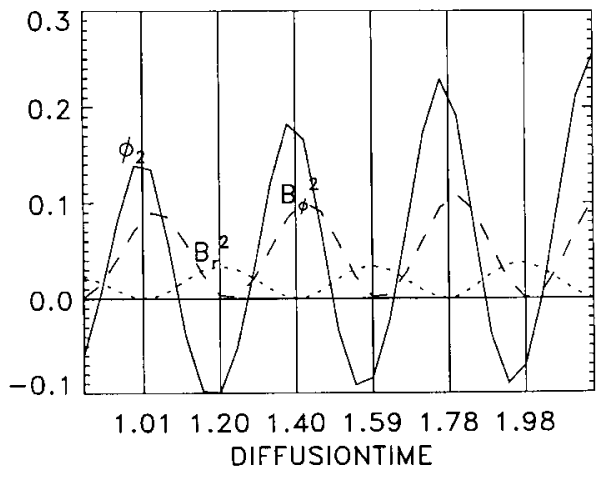

Fig. 7. Time series for $\phi_{2}(1)$. The positive (negative) sign indicates a magnetic-induced prolate (oblate) shape of the star. Note that the star is prolate during the maximum of the toroidal field energy and it is oblate during the maximum of the poloidal field energy. In the time average the star is prolate. The amplitude of the effect belongs to the arbitrary magnetic amplitude of the cycle shown in Fig. 6.

If we expand the potential up to the second order and substitute it into the relationship $\rho=\rho(\phi)$, we find

$$
\begin{aligned}
\rho(r, \mu) & =\rho\left[\phi_{0}(r)+\phi_{2}(r) P_{2}(\mu)\right] \\
& =\rho\left[\phi_{0}(r)\right]+\left(\frac{\mathrm{d} \rho}{\mathrm{d} \phi}\right)_{r} \phi_{2}(r) P_{2}(\mu),
\end{aligned}
$$

neglecting higher-order terms. If we compare Eq. (A.5) with the expansion of $\rho$ up to the second order, we immediately find

$\rho_{2}(r)=\left(\frac{\mathrm{d} \rho}{\mathrm{d} \phi}\right)_{r} \phi_{2}(r)$,

hence

$\rho_{2}(r)=\frac{[\rho(\phi)]^{2-\gamma}}{K \gamma} \phi_{2}(r)$

At the outer boundary of the configuration, i.e. for $r \rightarrow R^{-}$

$\rho \rightarrow 0, \quad \phi_{2}(r) \rightarrow \phi_{2}(R)=-3 G(M / R) J_{2}$.

Therefore, the outer boundary condition for the second order perturbation of the density is

$\rho_{2}(R)=0$.

\section{References}

Alexander, D. R., \& Ferguson, J. W. 1994, ApJ, 385, 621

Applegate, J. H. 1992, ApJ, 230, 815

Baryshnikova, Y. S., \& Shukurov, A. M. 1987, Astron. Nachr., 308, 89

Brandenburg, A., Tuominen, I., Nordlund, A., Pulkkinen, P., \& Stein, R. F. 1990, A\&A, 232, 277

Caughlan, G. R., \& Fowler, W. A. 1988, Atomic Data Nucl. Data Tables, 40, 283

DeCampli, W. M., \& Baliunas, S. L. 1979, ApJ, 230, 815

Elstner, D., Rüdiger, G., \& Schultz, M. 1996, A\&A, 306, 740

Ferraro, V. C. A. 1954, ApJ, 119, 407

Ferrière, K. 1992, ApJ, 391, 188

Guinan, E. F., \& Gimenez, A. 1993, Magnetic activity in close binaries, in The Realm of Interacting Binary Stars, ed. J. Sahade et al. (Kluwer), 95

Hall, D. S. 1976, in Multiple Periodic Variable Stars, ed. W. S. Fitch, IAU Coll., 29, 51 
Hall, D. S. 1989, Space Sci. Rev., 50, 219

Hall, D. S. 1990, Period changes and magnetic cycles, in Active close binaries, ed. C. Ibanoglu (Kluwer), 95

Iglesias, C. A., \& Rogers, F. J. 1996, ApJ, 464, 943

Kichatinov, L. L., \& Rüdiger, G. 1992, A\&A, 260, 494

Kippenhahn, R., Weigert, A., \& Hofmeister, E. 1967, Meth. Comp. Phys., 7, 129

Krause, F., \& Meinel, R. 1988, Geophys. Astrophys. Fluid Dyn., 43, 95

Lanza, A. F., \& Rodonò, M. 1999, A\&A, 349, 887

Lanza, A. F., Rodonò, M., \& Rosner, R. 1998, MNRAS, 296, 893

Linsky, J. L. 1988, Active late-type stars, in Multiwavelength Astrophysics, ed. F. Cordova (Cambridge Univ. Press), 49

Malkus, W. V. R., \& Proctor, M. R. E. 1975, J. Fluid Mech., 67, 417

Matese, J. J., \& Whitmire, D. P. 1983, A\&A, 117, L7

Mihalas, D., Hummer, D. G., Mihalas, B. W., \& Däppen, W. 1990, ApJ, 350, 300

Moss, D., \& Brandenburg, A. 1995, Geophys. Astrophys. Fluid Dyn., 80,229
Rädler, K.-H., \& Bräuer, H.-J. 1987, Astron. Nachr., 308, 101

Rodonò, M. 1992, The Rs-Canum stars, in Evolutionary Processes in Interacting Binary Stars, ed. Y. Kondo et al., IAU Symp. 151 (Kluwer), 71

Rogers, F. J., Swenson, F. J., \& Iglesias, C. A. 1996, ApJ, 456, 902

Rüdiger, G., \& Elstner, D. 1994, A\&A, 281, 46

Rüdiger, G., \& Kitchatinov, L. L. 1993, A\&A, 269, 581

Rüdiger, G., Elstner, D., \& Ossendrijver, M. 2002, A\&A, in preparation

Schubert, G., \& Zhang, K. 2000, ApJ, 532, 149

Schüssler, M. 1981, A\&A, 94, L17

Simon, V. 1999, A\&AS, 134, 1

Strassmeier, K. G., Hall, D. S., Fekel, F. C., \& Scheck, M. 1993, A\&AS, 100, 173

Weisshaar, E. 1982, Geophys. Astrophys. Fluid Dyn., 21, 285

Yoshimura, H. 1981, ApJ, 247, 1102

Zahn, J. P. 1989, A\&A, 220, 112

Zavala, R. T., McNamara, B. J., Harrison, T. E., et al. 2002, AJ, 123, 450 